\title{
Optical Interferometry from the Antarctic
}

\author{
Peter Tuthill \\ Sydney Institute for Astronomy, \\ School of Physics \\ University of Sydney \\ N.S.W. 2006 Australia \\ email: p.tuthill@physics.usyd.edu.au
}

\begin{abstract}
The unique atmospheric conditions which pertain in the high Antarctic plateau offer dramatic gains for many areas of Astrophysics. Optical Interferometry is among the most technologically demanding branches of modern instrumentation, and furthermore, is one which is most strongly limited by the stability of the atmosphere at the observatory site. The long-term potential for spectacular gains by implementing an interferometer on the high Antarctic plateau are presented.
\end{abstract}

Keywords. long baseline optical interferometry

\section{Introduction}

Many of the most topical astrophysical research frontiers entail observation of matter in the close environment of stars or highly luminous cores. Studies of exo-planets, star formation, stellar winds and active galactic nuclei are all limited by the extreme dynamic ranges, high angular resolutions and high measurement precision needed to discriminate the faint signals against the glare of a luminous central core. These considerations drive optical designs towards an interferometer, however at mid-latitude sites there is a heavy penalty from the seeing in the turbulent atmosphere.

Quantifying just how strong this penalty will be depends in detail on the specifics of the experiment, however interferometer performance will in general be strongly sensitive to all three fundamental seeing parameters: $r_{0}$ (spatial), $t_{0}$ (temporal) and $\theta_{0}$ (angular) coherence properties of the incoming wavefront. One mental construct to crudely quantify a site's seeing metric is to consider a hyper-volume consisting of the product of these three numbers - the larger this volume the better - but even such a multiplicative scaling probably under-represents the importance of good seeing. As most adaptive opticians know, when the seeing is sufficiently bad, it is better to just do something else with the telescope: one gets far less than half the science when fighting two arcsecond seeing compared to one arcsecond seeing.

From the Antarctic plateau, all three of these atmospheric properties attain their most favourable values on the surface of the Earth, as described in the opening session of this conference. For many crucial observational schemes such as nulling interferometry or precision astrometric interferometry, the dramatically improved conditions will result in orders of magnitude increase in sensitivity on top of gains already available from the extreme low temperature and water vapour.

The potential for Antarctic interferometry, enabled by such conditions, is fundamentally different from that at a conventional site. An Antarctic plateau interferometer could rival the immensely more expensive space missions to what many regard as the most important keystone project in modern astronomy: the characterization of exo-planets and detection of objects down to one earth-mass. Furthermore, a wealth of other stellar 
astrophysics can be addressed by such a device. Observation of disks around young stellar objects should reveal substructure such as spiral density waves and gap clearing due to planet growth. Mass loss phenomena in evolved stars, and substructure such as disks and jets within dusty compact microquasars, will be within reach of imaging observations. Studies of fundamental stellar properties - sizes, effective temperatures, distances and (using binary stars) masses - will mean that almost every branch of stellar astronomy will benefit.

\section{Optical interferometry prospects: a big picture view}

The field of long-baseline optical interferometry presently finds itself at something of a crossroads. Interest in the long-dormant field of high resolution imaging was rekindled worldwide by the dramatic success of the Narrabri Stellar Intensity Interferometer of Hanbury Brown and Twiss in the late 1960's to early 1970's (Hanbury Brown et al. 1967a; Hanbury Brown et al. 1967b; Hanbury Brown et al. 1974). There followed a flurry of prototypes and pathfinders in the 1980's and what can be justifiably described as a first major generation of dedicated science interferometers, mostly constructed in the decade leading up to the turn of the century. Another decade on, and we now find that the majority of these projects (more than half a dozen) have run their course and now been mothballed (e.g. KI, MIRA) or decommissioned (e.g. PTI, IOTA, GI2T). Notably, the survivors have been the ones with the more ambitious basic architecture (larger apertures; longer baselines; more telescope stations) which now boast high and still rising science output. In particular, the VLTI dominates the Southern, and the CHARA array the Northern skies at milli-arcsecond resolution; both instruments boast a rich arsenal of science instruments capable of a variety of observational modes.

However, the emergence of what might be described as a "second generation" of interferometers which build upon the successes of the first is not at all apparent. The only major new optical interferometric facility nearing science readiness is the LBT Interferometer whose novel architecture may well deliver unique science reach, but which seems more accurately described as the first coherent-light focus of an ELT rather than a long-baseline interferometer. The hope for significant new interferometer projects in the present decade rests with MROI and a major upgrade to NPOI; both with an uncertain (at the time of writing) funding outlook. Important progress is of course being made with new instrument subsystems such as the ambitious GRAVITY beam combiner at VLTI. Few proposals for new-generation interferometers of any real scope and ambition have been advanced; none seems to have attracted any significant following.

Although such a bleak picture might seem discouraging, the unquestioned potential for the basic physics of interferometry to deliver unique and critical knowledge remains. In particular, space missions (SIM, TPF, Darwin) to detect and image exoplanets around the stars in the solar neighbourhood and to characterize their basic properties have been pushed well into the future by funding constraints. However, the drive to accomplish the science remains strong and will only become more pressing as new discoveries continue to flesh out the statistical prevalence of exoplanets.

As has been pointed out before, the Antarctic provides a near space-analogue in many key respects and is available for the prototyping and commissioning of testbeds at a fraction of the price and complexity of a spacecraft. Maintaining ongoing efforts to advance the field and inform the design of tomorrow's audacious space missions targeting exoplanetary characterization is of critical importance. Wherever such spacecraft require the very ultimate in angular resolution, then the basic physics of detection will always drive 
designs towards the principles of interferometry. This will only lend weight beyond the direct science benefits outlined here to maintain a vibrant research community pushing the boundaries of these techniques.

\section{Architectures for optical interferometry}

Early experiments and interferometer prototypes typically recorded quite simple visibility (or $v^{2}$ ) amplitude information from only two telescopes over a single spectral bandpass. Such data can be recovered with a correspondingly simple beam combiner: at its most basic a beamsplitter to combine the beams and a pair of photodetectors to record the starlight fringes. However as the field has flourished, the capabilities of the instruments and the complexity of the data forms recovered has proliferated. There are now a large number of variations of interferometer design which target specific science niches. Each can make quite different demands on the basic instrumentation and infrastructure, and when a concept design is transferred to the high Antarctic plateau, each might realize gains or challenges from quite distinct aspects of the working environment.

Major interferometers at mid-latitude sites such as CHARA and VLTI have migrated towards a model similar to classical research observatories in which the large infrastructure such as telescopes and delay lines is kept as generally applicable as possible, and a variety of more specialized instruments are available to accept the starlight at the final step and deliver scientific results. Such a "switchyard" approach which allows a variety of science to be produced is unlikely to be profitable for Antarctic deployment due to its extra complexity and risk. The first major hurdle in planning an Antarctic Interferometer is therefore deciding upon the science goal and consequently the architecture do deliver it. Selecting the best match comes down to: (1) delivery of unique, world-leading science; (2) the instrument which exploits the unique conditions to greatest advantage over competitors; and (3) is most robust and reliable to operate with minimal support in the challenging environment. Some initial discusison of the trade-offs involved is given below as the merits of several plausible architectures are debated, although it is well beyond the scope of the present work to arrive at any quantitative conclusions. The interested reader is strongly encouraged to seek out the Arena Roadmap (2010) which goes into many key issues in far more detail than is possible here, and in particular the results of Working Group 3 led by Vincent Coude du Foresto.

\subsection{A narrow-angle astrometric interferometer}

If we assume that the physical layout of telescopes in an array can be very well characterized, then the trajectory of the interference fringe envelope generated by each baseline as a function of time can be used to extract very precise astrometric data recording the position of the stellar target. Repeated measurements covering many stars can generate an accurate all-sky wide-angle astrometric catalog (e.g. NPOI see Hutter et al. 1998), although the precision with which the effective baseline can be measured does limit the final accuracy.

One way to dramatically boost the precision while keeping the baseline metrology requirements relatively modest is to implement a narrow angle astrometric mode in which the interferometer switches between observations of relatively close $(\lesssim$ few arcsecond) binary stars. Such an observing mode was pioneered with the PHASES instrument at the Palomar Testbed Interferometer (Muterspaugh et al. 2010), while several instruments 
worldwide such as GRAVITY and PRIMA at the VLTI and MUSCA at SUSI all employ a set of similar basic principles.

There are several specific advantages such an interferometer design would gain from Antarctic deployment. In particular, the fundamental limitation to the astrometric measurement process is an extremely strong function of the isoplanatic patch size (Lloyd et al. 2003). Of all the unique properties of Antarctic plateau seeing, perhaps this is the one which is most exceptional due to the concentration of the atmospheric turbulence in the near-field at the boundary layer. Furthermore, telescope apertures and baselines can be comparatively modest.

Mitigating against this design are several practical factors. Astrometric modes have proven to be relatively difficult and complex to implement at mid-latitude interferometers, and none has yet approached the fundamental performance limitation set by the atmosphere. Furthermore, any such instrument will face severe near-term competition from the Gaia mission which will achieve $\lesssim 20 \mu$ arcsec precision over a relatively deep whole-sky survey.

\subsection{A nulling interferometer}

An interferometric analog of a Coronagraph, Nulling interferometer designs hold out the promise of rejecting the overwhelming glare from a bright central star yet allowing the study of faint circumstellar material or structures in the immediate vicinity. The original idea of placing a destructive interference fringe to cancel the star dates back to Bracewell (1978). The first and most ambitious modern separate telescope nulling instrument was operated as a part of the Keck interferometer (Serabyn et al. 2012) which employed a 4-beam nulling configuration delivering significantly deeper nulls than a simpler 2-beam configuration could achieve.

Obtaining reasonable null depths motivates a requirement for relatively good wavefront quality. In the absence of a high-order adaptive optics system (both complex and expensive), this wavefront quality is one factor that pushes nulling designs towards longer wavelengths in the mid-infrared. Fortunately, much of the most profitable astrophysics is also best studied in this waveband. Varying forms of dusty disks from exo-zodiacal clouds to debris disks all offer considerable advantage to longer wavelength study. Furthermore, the early stages of brown dwarf and planetary assembly are those in which the object is both hottest and brightest, with contrast ratios becoming dramatically less challenging as one moves from the visible to the mid-infrared.

Nulling interferometry stands to make very significant gains when implemented on the Antarctic plateau. Long-wavelength operation benefits from good atmospheric transparency (low water vapor), and more importantly, from the thermal environment. Typical interferometer optical trains may have more than 20 reflections bringing the starlight to the detector. Reducing the self-induced thermal radiation from the mirror train itself by exploiting the cold ambient conditions will already deliver a profound impact on the sensitivity of the device, before one factors in extra gains due to the excellent seeing. A scientifically competitive nuller would, however, require some reasonable scale of infrastructure with moderately large apertures $(\gtrsim 0.5 \mathrm{~m})$ and designs may require considerable engineering in order to exploit the good seeing above the boundary layer.

\subsection{An imaging/closure phase interferometer}

Among the commonest functionalities for a modern optical interferometer is to recover complex visibility data products. Such devices are the most direct descendents from 
detection schemes employed in the earliest devices. Unfortunately extreme measurement precision, well beyond anything yet demonstrated, would be required for such instruments to reveal the presence of high contrast companions by exploiting visibility amplitude data alone. Much emphasis and effort has instead been focused on observables extracted from the Fourier Phase. Although the phase itself is corrupted by the atmospheric seeing, observables such as differential phase and particularly the closure phase hold the promise of pushing detection thresholds into the planetary regime for systems which are young and bright.

Indeed, within the context of aperture masking interferometry, the use of closure phase methods to recover high contrast companions well into the planetary mass regime has become well established in recent years (Tuthill et al. 2006; Lloyd et al. 2006) and indeed has delivered the first direct detection of a planetary candidate at the epoch of formation (Huélamo et al. 2011).

Practical implementation of such methods is not strictly prescriptive of the interferometer architecture nor even the observing wavelength, although reasonable extrapolations of successful current experiments would most likely come up with a strawman near-infrared device with several $(4 \sim 6)$ telescopes of moderate $(\gtrsim 0.5 \mathrm{~m})$ aperture. Gains over mid-latitude sites would mainly arise from superior seeing, provided the boundary layer problems can be solved, although the potential to leverage still further thermal gains towards somewhat longer wavelengths in $\mathrm{L}$-band $(3.6 \mu \mathrm{m})$ is also attractive.

One potentially interesting variation to this design may be to explore the idea of a closure-phase nulling interferometer, as proposed by Chelli et al. (2009). This requires baselines sufficiently long to reach the first null of the stellar visibility function, which for nearby bright stars is of order $\sim 300 \mathrm{~m}$ or so.

\subsection{Reformulating the optical interferometer}

Optical interferometer designs from the 20th century relied heavily on precision control with precisely engineered components implemented in (as much as possible) a thermally and mechanically stable environment. Following the same recipe may be costly and difficult in an Antarctic plateau context, particularly so when the requirement to be elevated some $\sim 20 \mathrm{~m}$ above the ice to avoid the boundary layer seeing.

It is therefore interesting to ask whether all this concrete and precision-milled steel is really necessary to perform the basic functionality of an interferometer? One telling point to note is that for most functioning instruments, despite the money spent to deliver high degrees of inherent mechanical stability, it remains a part of normal procedure to search over some relatively wide interval for the fringes - often many millimetres. Small changes in optical alignment, together with errors introduced at moving parts and thermal/mechanical drifts, conspire to create pathlength imbalances.

I therefore propose a shift in the underlying philosophy of interferometer design. Rather than attempting to control optical surfaces and components to the micron level, instead we merely perform metrology adequate to characterize these paths in real time. Such information can be employed to drive a short-throw delay line to compensate for pathlength excursions which should be relatively slow (if care is taken to minimize mechanical vibration in the instrument). Tabulated below is a straw-man design which reformulates an interferometer along these principles. 
Classical Interferometer Subsystem |Reformulation for Antarctica

Light Collecting
Typically afocal telescopes or siderostats mounted on very stable concrete piers or (for designs with movable telescopes) kinematic mounts at fixed telescope base stations.

\section{Beam Transport}

Convey light collected over an array spanning hundreds of metres to a common point. Typically evacuated steel pipes with an internal embedded chain of relay mirrors.

\section{Delay Lines}

Starlight must be pathlength matched to micron precision with a varying delay according to the projected geometry of each interferometer baseline onto the sky. This is done with motorized carriages running on precision steel rails under laser metrology control. Ideally, the entire delay line is implemented in a vacuum.

\section{Beam Combination}

Beam combiners and all the optical processing that goes with them is usually achieved with one (or more) optical table of lenses, mirrors and beamsplitters.

\section{Light Collecting}

The basic optics can be similar, although mounted on towers to rise above the inversion layer. Located to sub-mm accuracy with some form of metrology grid (several technical solutions exist).

\section{Beam Transport}

Use pre-matched lengths of optical fiber deployed to each telescope. For a continually moving telescope design (see next point) then dragging a fiber cable may be a viable option.

\section{Delay Lines}

Possibly delay lines running in a tunnel with a refrozen flat ice floor. More radically, implement continually moving telescopes on an ice plane thereby removing the need for any separate delay line (and performing the delay correction effectively in vacuum).

\section{Beam Combination}

Accomplish all optical processing functionality in integrated optics chips. These are ideal for challenging environments: hermetically sealed, alignment-free, and highly robust.

\section{Conclusions}

A discussion paper on prospects and designs for a future Antarctic interferometer has been presented. Several basic architectures and specific science cases seem promising enough to merit further exploration; in particular a mid-infrared nulling interferometer and a near-infrared imaging/closure phase instrument. From the perspective of fundamental physical principles, interferometry presents the natural way to obtain information on the finest possible angular scales and so reveal the physics of processes such as stellar and planetary assembly which occur on such a remote stage that milli-arcsecond resolution is required. The high Antarctic plateau offers conditions dramatically superior to any other known terrestrial site for this endeavor, delivering order-of-magnitude gains to instruments operating at fundamental noise limits set by the turbulent atmosphere. The author hopes, therefore, that the present dearth of major new activity in interferometry might be likened to a saturated solution of science potential with ever increasing concentration. All it takes is one crystal - perhaps an Antarctic snowflake - to suddenly cause a flurry of new activity to precipitate. 


\section{References}

ARENA Roadmap 2010. Available online at arena.oca.edu

Bracewell, R. N. 1978, Nature, 274, 780

Chelli, A., Duvert, G., Malbet, F., \& Kern, P. 2009, A\&A, 498, 321

Hanbury Brown, R., Davis, J., Allen, L. R., \& Rome, J. M. 1967, MNRAS, 137, 393

Hanbury Brown, R., Davis, J., \& Allen, L. R. 1967, MNRAS, 137, 375

Hanbury Brown, R., Davis, J., Lake, R. J. W., \& Thompson, R. J. 1974, MNRAS, 167, 475

Huélamo, N., Lacour, S., Tuthill, P., et al. 2011, A\&A, 528, L7

Hutter, D. J., Elias, N. M., \& Hummel, C. A. 1998, Proc. SPIE, 3350, 452

Lloyd, J. P., Martinache, F., Ireland, M. J., et al. 2006, ApJL, 650, L131

Lloyd, J. P., Lane, B. F., Swain, M. R., et al. 2003, Proc. SPIE, 5170, 193

Muterspaugh, M. W., Lane, B. F., Kulkarni, S. R., et al. 2010, AJ, 140, 1579

Serabyn, E., Mennesson, B., Colavita, M. M., Koresko, C., \& Kuchner, M. J. 2012, ApJ, 748, 55

Tuthill, P., Lloyd, J., Ireland, M., et al. 2006, Proc. SPIE, 6272, 